\title{
Design and Realization of a Certain Type of Radio Detecting Repair and Training Platform
}

\author{
Shao Zhichao ${ }^{1, a}$, Tu Jianhua, a , Shu Chang ${ }^{1, a}$, Liu Junxian ${ }^{1, a}$, Zhou Peng $^{1, a}$, \\ Han Hongliang ${ }^{1, a}$ \\ ${ }^{1}$ Wuhan Mechanical Technology College, WuHan, HuBei, 430075, China \\ ashaozhichao@sina.com
}

Keywords: Detecting and maintenance training platform; AF signal; RF signal; Adapter

\begin{abstract}
For the purpose of testing, detecting and maintaining a certain type of radio, a detection and maintenance training platform is researched and invented. The paper introduces the design and realization of the platform, which consists of two parts: the adapter and the host computer software. Practice shows that the platform, running stably and reliably, can greatly improve the testing, inspection and maintenance of the machine and the circuit board of the radio, so that provides convenience for teaching the radio.
\end{abstract}

\section{Introduction}

The certain type of radio is a high-tech electronic information equipment with complex structure and high price.As an important wireless communication device in command and control systems, the radio's condition directly determines the operation of the systems. At present, there is a lot of inconvenience in testing and maintenance training of the radio. For example, when using a dedicated radio testing equipment to examine the whole radio, the result only shows whether the receiver and transmitter is normal or not, but lacks the specific testing indicators value; and board-level testing is blank. To address this problem, we developed a type of radio detection and maintenance training platform, which can solve the difficulties in radio detection and maintenance training, and realize the maintenance training for the whole machine and the circuit board of the certain type of radio.

\section{Platform Introduction}

The radio testing and maintenance training platform can be used for machine automated testing, circuit board automated testing, circuit board detecting, automatic test and diagnostics on the radio and testing indicator giving of the certain type of radio. The platform overcomes the difficulties in detecting performance parameters while working and presenting working principle by the traditional teaching, leading out the circuit board interface data that need testing and test them. Combined with the working process of the circuit board, it gives visual display teaching and training, providing a good platform for radio detection and maintenance training. The testing and maintenance platform has the following features.

- Radio's transmitter test

The platform can test the performance indicator of the radio transmitter. As the modulation of the Radio is AM, the performance indicators that can be tested mainly refer to RF output power (the high and low power of single-sideband), transmitter power, the unwanted sideband suppression and carrier frequency, audio modulation characteristics, spurious narrowband RF components and the maximum frequency error.

- Radio's receiver test

The platform can test the performance indicator of the radio receiver. The indicators that can be tested include receiver power consumption, sensitivity, selectivity, audio response, the total distortion factor and the audio output.

- Automatic test of the radio circuit board 
Based on the testing of the machine performance indicator, automatic test of the radio circuit board can be operated. Through interaction of the host computer, the control adapter provides a working environment for the circuit board to be tested, so that automated test gets completed in a normal working condition. The test results are real-timely displayed on the screen.

- Radio circuit board test

Under automatic test condition, the radio circuit board can be tested through the test interface (leads out the data of the radio circuit board interface that need to be detected) with oscilloscope and other common test instruments for circuit board testing and maintenance training.

\section{Design of platform hardware}

The platform hardware includes host computer, radio test adapter, the measured radio and the measured radio circuit board, shown in Figure 1.

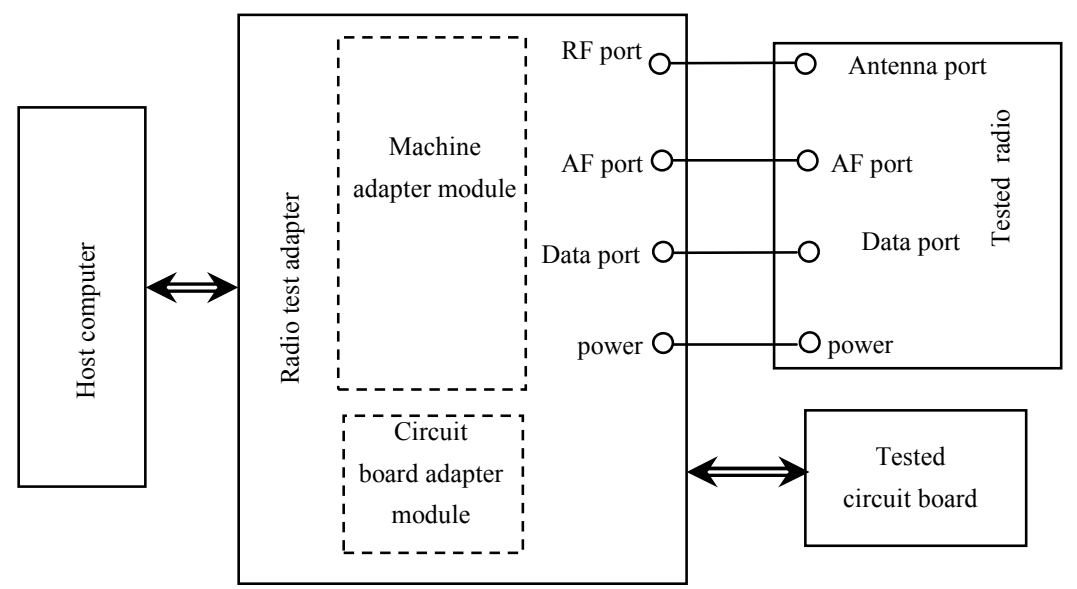

Figure 1 Block diagram of automatic testing module hardware

Consisting of mainframe, LCD monitor, keyboard and mouse, the host computer controls the radio's test adapter through the software that the mainframe runs, as well as collects and processes the output signal of the radio machine and tested circuit board, with the results shown on the display screen in real time.

The radio's testing adapter includes a radio machine adapter module and a circuit board adapter module. The Machine adapter is connected with the antenna port, audio port, data port and power port of measured radio through the RF port, audio port, data port and power port, providing RF signal, audio signal, control signal and power signal for the radio. Circuit board adapter offers working environment for the radio circuit board to be tested, so that it can be in normal working condition.

The measured radio is the radio's transceiver host. The tested circuit board includes the radio's power control unit, M/ LF unit, RF unit, amplifier unit, the frequency of co-unit, filter unit, day tune unit etc.

- Design of the machine adapter module

The design of the radio's machine test is as follows: on measuring the performance indicators of the transmitter, the adapter's audio signal generating circuit imports into the radio audio signal through the testing device and turns on the PTT switch to start the transmitter. RF signal testing circuit receives and tests the frequency modulation signal (AM) emitted by the radio station, and analyzes the internal information of the RF signal to get the transmitter's indicator parameters of the tested equipment. On testing the receiver's performance indicator, the adapter's RF signal generating circuit imports into the radio modulated RF signal. The audio signal testing components receive the audio output signal emitted by the radio, and demodulate and test the signal to get the index of the radio receiver. 
The whole adapter is a bridge between the host computer and the tested station, including the RF module and audio module, with each module containing circuits of signal generation and signal analysis. The RF and audio signal generating circuit is responsible for RF signal generating, audio signal generating, and the measured radio control. RF and audio signal testing circuit responses to the signal receiving, processing and collection. Its block diagram is shown in Figure 2.

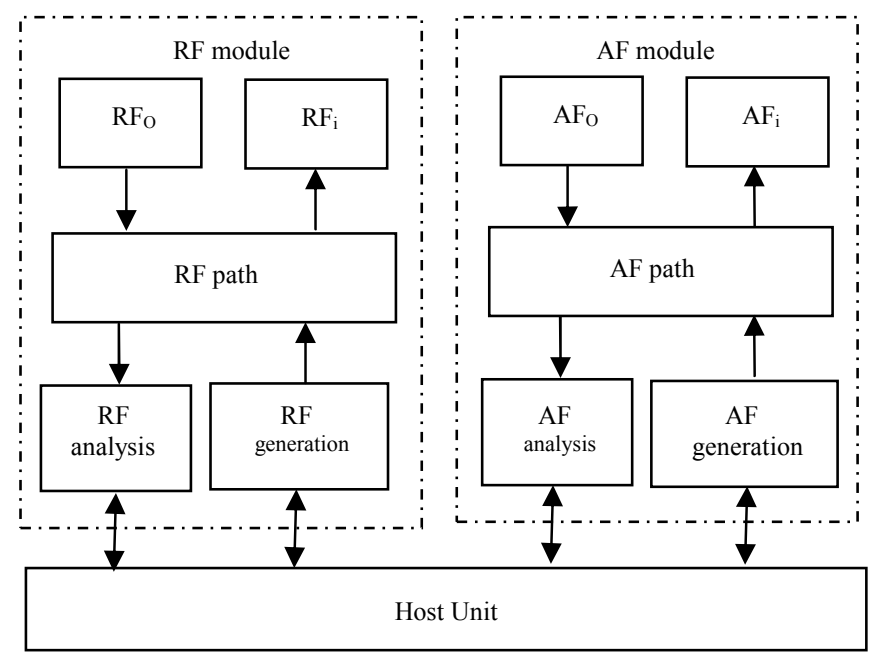

Figure 2 Block diagram of whole adapter module

- Design of circuit board adapter module

The design of automated testing of the radio's circuit board is as follows: the host computer is connected to adapter and data acquisition card via respectively RS-232C serial port and PCI bus, shown in the block diagram of Figure 3 below.

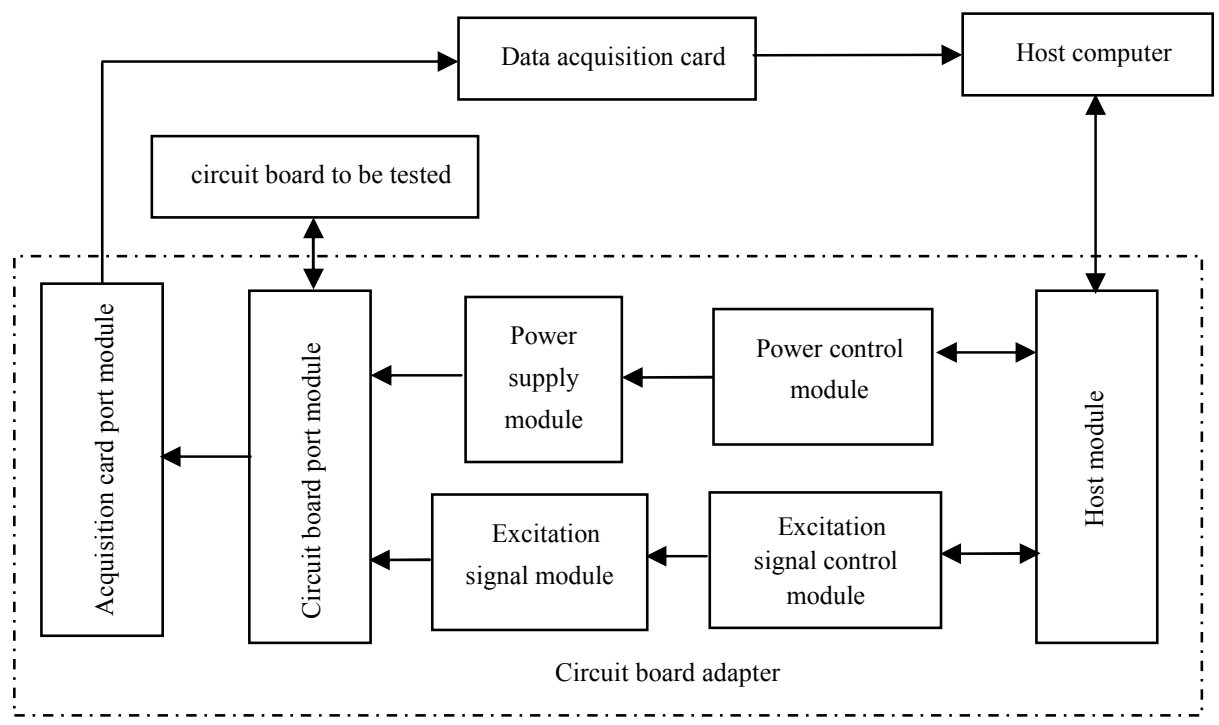

Figure 3 Block diagram of circuit board adapter module

Through RS-232C serial port, the host computer controls adapter to provide working environment for the circuit board to be tested. At the same time, through the main control module, it switches the tested circuit board among different working conditions. The output signals of the tested circuit board are collected and processed by the data acquisition card and then are sent to the host computer through the PCI bus. The host computer software handle and analyze the received circuit board testing data, and compare them with the standard signal of circuit board, and display the results on the monitor in real-time, therefore automated testing of the radio circuit board is achieved. On automatically detecting a circuit board, general-purpose testing instrument like oscilloscopes can be used for manual inspection training. 
Circuit board adapter is made up of power supply module, power control module, excitation signal module, excitation signal control module, circuit board interface module and acquisition card interface module.

- Power supply module

The power supply module, on the one hand, produces the required voltage for each working circuit module of the adapter, on the other hand, offers working voltage and control signals for the circuit board to be tested. The Power is AC $220 \mathrm{~V}$ voltage, and changes from AC to DC $(+24 \mathrm{~V})$ through switching power supply NES-150-24. $+24 \mathrm{~V}$ voltage generates DC voltage such as $+5 \mathrm{~V},+10 \mathrm{~V},+12$ $\mathrm{V},+15 \mathrm{~V}$ through three-terminal regulator chips of 7805,7810 etc. providing power and control signals for the adapter circuit module and circuit board to be tested.

- Excitation signal module

The excitation signal module generates various excitation signals which are required by circuit board to be tested, including RF signal and audio signal, achieved by using DDS (Direct Digital Frequency Synthesizer) technology. Different DDS module is adopted depending on the frequency of the excitation signal.

- RF signal generator circuit

RF signal generating circuit excitation is the hard point in signal generating circuit, used to generate the required RF signals when testing the radio's receiver. The RF signals that the radio's receiver can receive are the AM signals with the carrier frequency between 1.6 and $29.9999 \mathrm{MHz}$ and frequency interval being $100 \mathrm{~Hz}$. In order to export highly stable analog RF signals of different carrier frequency, the RF signal generating circuit has to adopt a digital phase-locked frequency synthesizer which is composed of phase-locked loop IC TSA5526 and voltage-controlled oscillator ROS-200. The composition is shown in Figure 4.

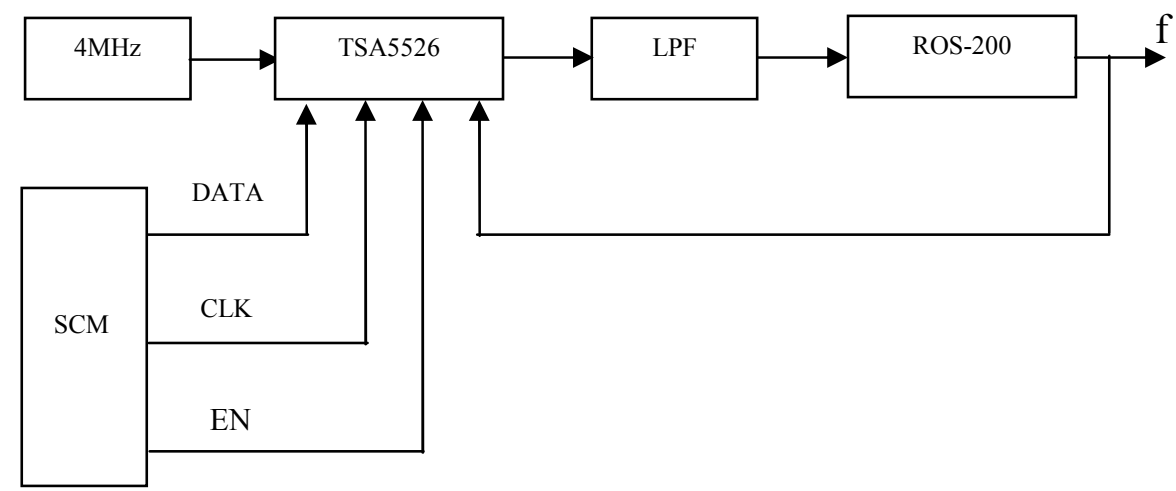

Figure 4 Block diagram of RF signal generating circuit

- Audio signal generating circuit

The audio signal generating circuit is used to generate the desired audio signal when testing the radio transmitter. According to the figures of the tested radio's audio signal, the circuit needs to generate audio signals with a frequency range of $1 \mathrm{KHz} \sim 520 \mathrm{KHz}$.

In order to export highly stable analog audio signals of different frequency, the audio signal generating circuit has to adopt the chip of AD7008 and DDS consisting of external circuits. DDS chip AD7008 can generate tone signals at the frequency range of $10 \mathrm{~Hz} \sim 10 \mathrm{MHz}$. the signals, after handling by the filter circuit and amplifier circuit, are sent to the standard signal end of the D / A chip. The control codes of the signals sent through the SCM are further processed in the D / A chip, and produce highly stable audio signals with controllable amplitude and frequency.

- Master module

According to the instructions sent by the host computer, the master module dominates the work of the power control module and the excitation signal control module, and then turns to control the power module and the excitation signal module to generate voltage and signal for the normal work of the circuit board to be tested. 
The state control circuit is an important part of the master module, mainly responsible for the control of the working state of radio. The software of the host computer through the RS-232C serial port control the master circuit, which controls the radio working state by using controlling signal (such as words PTT, message PTT, words sending-in, words receiving-out, etc.), so that achieving the conversion among the radio's working state like receiving, sending, words, press, upper sideband and lower sideband.

- Signal acquisition module

Signal acquisition module gets realized by using data acquisition card. Based on a USB, data acquisition card adopts NI's M Series data acquisition card NI-USB 6259,32 analog input, sampling rate of $125 \mathrm{MS} / \mathrm{s}$, 16-bit resolution; 4 analog outputs, 16 bit resolution; 48 digits I / O lines (8 of them are $1 \mathrm{MHz}$ hardware timing lines); 2-way 32-bit counter / timers, aimed for PWM, encoder, frequency counting. The acquisition card can meet the needs of collecting the radio's testing signals. The input end of the card is linked with radio's testing signals through the adapter. The circuit board testing signals enter the card, and the processing results for the collected signals are displayed on the monitor.

\section{Design of platform software}

\section{- Software}

Platform software is mainly responsible for controlling the radio's testing adapter via the host computer, and for processing the output signals of tested radio and circuit board collected by the data acquisition card, with the results displaying in real time on monitor.

On the human-computer interaction interface of the host computer software, we can choose to test the whole machine or the circuit board. The automatic testing module automatically control the radio's testing adapter to start the testing program, so that the radio or the circuit board is in the state of being tested. The data acquisition card automatically receives and processes the test signals for the radio or board, and the results are real-timely displayed on the monitor. The results include the radio's whole performance indicator, circuit board testing signal value and the normal or informal state of testing signals, so that performance testing and troubleshooting for the tested radio and tested circuit board can be carried out.

- software program

As the soul of the automatic testing module, the software system exerts a direct impact on the functionality and operability of testing. Software design includes human-computer interactive interface design and testing process design, developed with National Instruments (NI) LabVIEW software. The design of software process is as follows: according to the functional requirements and the features of hardware, and to ensure program portability and maintainability of the program, the software is designed with hierarchical structure and modular design. For improving testing efficiency, we optimize the testing process based on the characteristics of tested objects. For example, the receiver's audio output and sensitivity can be tested simultaneously, and the testing of transmitter's carrier power and frequency stability can be performed simultaneously.

When processing the test signals of the circuit board, the software first pretreats the testing signals of the circuit board, and then has classified calculation to the signals. If the signal type is DC voltage, Average DC / RMS function automatically calculates the size of the DC component of the signal; if the signal type is AC voltage, the Amplitude and Level function calculates the amplitude, maximum and minimum values of the signals; FFT Spectrum function carries out Fourier transform to the signals and calculates the frequency. Finally, the results of the testing signal processing are compared with the standard signal values in the database. Whether the calculated signal is within the scope of the standard signal given determines that the results showing is "normal" or "abnormal" , and the calculated and compared results of the testing signals are also displayed on the monitor. 


\section{Conclusion}

The testing and maintenance training platform can provide advanced training facilities and training methods for radio maintenance and support. The inspection and testing of the circuit board can especially facilitate the deeper learning of principle testing and maintenance training. After testing the computer software and hardware environment, the platform proves running stable, greatly improving the efficiency and level of implementation in practical teaching, and providing convenience for actual testing and maintenance training.

At present, this testing and maintenance training platform has been used in teaching practice. The using experience has shown that the platform has good effect. But it still has the following problems: First, the platform involves the communication between the host computer testing software and hardware platform, and at the same time, it has to real-timely collect and process large amounts of circuit board signal and displays and show the results, which to some extent, reduces the speed of the software. Second, the circuit board is sheltered in the shield cavity when it works in the radio, so the RF signals get suppressed. While when an adapter provides a working environment for the circuit board, the shielding cavity lacks, which results in slightly changes of RF signals of some key detection points on the board, though the normal work of the entire board is not affected. The further work of this paper is to solve the above two issues.

\section{References}

[1] Liu Cun, Modern Detection Techniques [M] Beijing: Mechanical Industry Press, 2005, pp.65-69.

[2] Zhou Yuwei, Automatic Detection Technology [M] Wuhan: Huazhong University of Science Press ,1995, pp.112 -116.

[3] Houbo Heng, Libo Cheng, 16 Microcomputer Principle and Interface Technology [M]. Xi'an: Electronic Science and Technology University Press ,1996, pp.79 -83.

[4] Mei Jinguo, Yang Zhiping, IC Application Design [M]. Xinyang: The First Aeronautical College Press ,2000, pp.124 -127.

[5] Yu Mengchang, Fundamentals of Digital Electronics Concise Guide [M] Beijing: Higher Education Press ,1999, pp.98 -104. 